\title{
The haptic perception of curvature
}

\author{
IAN E. GORDON and VICTORIA MORISON \\ University of Exeter, Exeter, England
}

\begin{abstract}
Ninety-two subjects, schoolchildren and undergraduate and postgraduate students, took part in a series of experiments on the haptic perception of curvature. A graded series of surfaces was produced using plano-convex lenses masked off to produce curved strips that could be explored without using arm movements. Thresholds were measured using the constant method and a staircase procedure. Experiments 1 and 2 yielded data on the absolute and difference thresholds for curvature. Experiment 3 demonstrated that the effective stimulus for curvature is represented by the overall gradient of a curved surface. Using this measure, it was shown that the present absolute thresholds for curvature are lower than those previously reported. In Experiment 4, absolute thresholds were compared using spherical and cylindrical curves: the results showed that, at least with the narrow strips used, the type of curvature does not exert a significant influence on performance. In Experiment 5, the subjective response to curvature was assessed using a rating procedure. Power functions are reported, although the relationship between stimuli and responses had a strong linear component. This suggests that haptically perceived curvature may be a metathetic rather than a prothetic continuum.
\end{abstract}

This report presents psychophysical data on the haptic perception of curved surfaces. The need for such data arose during an investigation of haptic detection of undulations in surfaces (Gordon \& Cooper, 1975). The majority of studies of the perception of curvature have used quite long surfaces. One obvious reason for this is that such surfaces are easier to produce. We thought it would be interesting to see how well people can perceive curvature when the surfaces are short and can be explored without gross movements of the hand and arm.

There are relatively few publications on the perception of curvature, and the most relevant of these can be described briefly. Crewdson and Zangwill (1940), Hunter (1954), and Rubin (1936) used similar procedures, and only one of these studies will be described. In Crewdson and Zangwill's investigation, a blind adult judged whether the edge of a $247-\mathrm{mm}$ flexible steel strip was straight or curved. The strip was supported at each end and its curvature manipulated by displacing the center. Detecting that the strip was not straight required approximately $2-\mathrm{mm}$ displacement of the center. Interestingly, some concave curvature was required for the strip to be judged straight.

In a more recent study, Davidson (1972) examined the performance of blind and sighted subjects as they judged the curvatures of the edges of $200-\mathrm{mm}$ strips. Four arc heights (the perpendicular distance from the midpoint of the arc's chord to the midpoint of the

\footnotetext{
This research was supported by a grant from the British Medical Research Council. We thank S. E. G. Lea for valuable advice. Address reprint requests to either author, Department of Psychology, Washington Singer Laboratories, University of Exeter, Exeter EX4 4QG, England.
}

arc) were presented in concave and convex orientations. Davidson's results consist of psychophysical data together with descriptions of the various scanning strategies employed by his subjects. Blind subjects tended to scan differently from sighted subjects and achieved lower absolute thresholds.

Davidson's results are interesting, and his apparatus and procedures appear well suited to his purpose. But his threshold data do not constitute an exhaustive account of the haptic perception of curvature. Only 4 deg of curvature were presented, the reliability of the psychophysical functions is not reported, and difference thresholds were not established. Most importantly, the use of long surfaces required extensive exploratory movements. As we shall show, lower thresholds can be obtained using shorter surfaces.

The aims of the present research were: (1) to measure absolute and difference thresholds for haptic detection of curvature, (2) to establish the effective stimulus for curvature, and (3) to discover the function relating the subjective rating of curvature and the objective curvature of surfaces.

\section{EXPERIMENT 1: THE ABSOLUTE THRESHOLD FOR CURVATURE}

\begin{abstract}
Method
Apparatus. The test surfaces were constructed from a set of 60-mm-diam plano-convex lenses, each with a $2.0-\mathrm{mm}$ step edge. The lenses ranged from 0 D (the "blank") to $3.0 \mathrm{D}$ in .125-D steps. The curved surface of each lens was masked off with adhesive paper to leave a $10 \times 20 \mathrm{~mm}$ central srip.

Subjects. Two groups of subjects were tested: (1) 10 fifth-form schoolchildren from a local school, with a mean age of 16.8 years,
\end{abstract}


and (2) 17 psychology undergraduates, with a mean age of 19.9 years.

Procedure. A two-response forced-choice version of the method of constant stimuli was used. A trial consisted of 20 paired presentations of the blank surface and one presentation of the curved surfaces, presented in random order. Subjects felt each surface by moving the tip of the second finger of the preferred hand twice across the central strip. During this haptic exploration, the forearm remained stationary while the wrist and fingers moved. The edge of the masking paper formed a prominent tactile boundary to the strip. Speed of movement was not controlled, but the subjects' average time for the double traverse was approximately $1 \mathrm{sec}$. Prior to each trial, talcum powder was applied to the subject's finger to reduce friction between the skin and the glass surface. Pilot studies had shown that giving feedback at the end of each trial exerted no effects upon performance at this task, so none was given during the main experiment.

\section{Results}

The proportions of correct detections per trial were converted to $z$ scores. The relationship between $z$ scores and the base-to-peak heights of the test surfaces was analyzed by linear regression from which the absolute thresholds were derived. Results for which $\mathrm{r}^{2}$, the index of goodness of fit of the regression, was not significant by $t$ test $(p<.05)$ were excluded. This led to the loss of seven subjects in the experiment, four schoolchildren and three undergraduates. Further analysis revealed that there is no significant difference between the thresholds of the schoolchildren and the undergraduates, which are summarized in Table 1.

The ability to detect that a surface has some curvature is impressive. The average of the threshold data from Experiment 1 is equivalent to detecting a curve with a base-to-peak height of only $.09 \mathrm{~mm}$ in a $20-\mathrm{mm}$ strip. Rightly or wrongly, we were surprised by this level of performance.

\section{EXPERIMENT 2: THE DIFFERENCE THRESHOLD FOR CURVATURE}

\section{Method}

The apparatus and procedure were as in Experiment 1 except that the blank lens was replaced by a curved standard surface with a base-to-peak height of $.12 \mathrm{~mm}$ (Condition A) or $.14 \mathrm{~mm}$ (Condition $B$ ). The subject's task on each presentation was to decide which of a pair of surfaces, presented sequentially in random order, had the greater curvature.

Table 1

Thresholds for Curvature (Base-to-Peak Heights in Millimeters): Experiments 1 and 2

\begin{tabular}{lcc}
\hline & Mean & SD \\
\hline Experiment 1: Absolute Threshold & \\
Schoolchildren & .08 & .02 \\
Undergraduates & .09 & .03 \\
Overall & .09 & .03 \\
\multicolumn{1}{c}{ Experiment 2: Difference Threshold } & \\
Condition A (Standard $=.12 \mathrm{~mm}$ ) & .10 & .02 \\
Condition B (Standard $=.14 \mathrm{~mm})$ & .12 & .04 \\
\hline
\end{tabular}

Subjects. The subjects were psychology undergraduates, with a mean age of 20 years. Eight were tested under Condition $A$, and seven were tested under Condition $B$.

\section{Results}

Analysis of data was as in Experiment 1. One subject's scores were excluded from the data summarized in Table 1 because of a nonsignificant fit in the regression analysis. Difference thresholds were derived from individual equations, and the means are shown in Table 1, from which it can be seen that the differential limens (DLs) for curvature are similar for the two standard stimuli.

Once again, we find the subjects' haptic performance quite impressive. The average difference thresholds in Experiment 2 represent an ability to discriminate between two curved $20-\mathrm{mm}$ surfaces when their base-to-peak heights differ by as little as $.11 \mathrm{~mm}$.

\section{EXPERIMENT 3: THE EFFECTIVE STIMULUS FOR THE PERCEPTION OF CURVATURE}

In the first two experiments, we used base-to-peak height as an objective measure of spherically curved surfaces. The fact that performance in Experiments 1 and 2 tended to vary monotonically with base-topeak height confirmed the usefulness of this measure. There are, however, at least two other aspects of spherical curvature, either of which could better represent the effective stimulus. These are the radius of curvature of the surface and a measure that, by including the length as well as the height of the curve, represents the gradient of the surface. Experiment 3 was designed to compare these alternatives and the base-to-peak height measure.

\section{Method}

The experiment was a simple extension of Experiment 1, with the introduction of two additional surface lengths: 30 and $40 \mathrm{~mm}$.

Subjects. The 12 subjects were psychology undergraduate and postgraduate students from the Exeter department, with a mean age of 25 years. Six were tested in each condition.

\section{Results}

Threshold calculations were as in Experiment 1. It was not necessary to exclude any subject's data from this analysis. The absolute thresholds recorded, using the two curve lengths, are summarized in Table 2, together with the overall average threshold from Experiment 1 recorded under very similar conditions. Preliminary inspection of threshold data using three curve lengths revealed that, although thresholds vary monotonically with base-to-peak height and inversely with radius of curvature (the two measures being related), neither measure alone provides an exhaustive description of the effective stimulus for curvature. This conclusion stems directly from two find- 
Table 2

Absolute Curvature Thresholds for Three Surface Lengths: Experiments 1 and 3

\begin{tabular}{|c|c|c|c|c|c|}
\hline \multirow{3}{*}{$\begin{array}{l}\text { Experi- } \\
\text { ment }\end{array}$} & \multirow{3}{*}{$\begin{array}{l}\text { Length of } \\
\text { Surface }\end{array}$} & \multicolumn{4}{|c|}{ Threshold } \\
\hline & & \multicolumn{2}{|c|}{$\begin{array}{c}\text { Base-to-Peak } \\
\text { Height }\end{array}$} & \multicolumn{2}{|c|}{$\begin{array}{l}\text { Base-to-Peak Height } \\
1 / 2 \text { Length }\end{array}$} \\
\hline & & Mean & $\mathrm{SD}$ & Mean & $\mathrm{SD}$ \\
\hline 1 & 20 & .09 & .03 & .009 & .003 \\
\hline 3 & 30 & .13 & .06 & .009 & .005 \\
\hline 3 & 40 & .18 & .05 & .009 & .003 \\
\hline
\end{tabular}

Note-Length and height are given in millimeters.

ings: (1) those lenses associated with threshold performance in one condition are not the same as those associated with threshold performance in other conditions, and, hence, radius of curvature cannot predict performance when different lengths of surface are employed; (2) thresholds, expressed in terms of baseto-peak height, are significantly different under the three conditions (Kruskal-Wallis $\mathrm{H}=12.0, \mathrm{p}<.01$ ).

When, however, a gradient measure is derived by dividing base-to-peak height by half the surface length, performances under the three conditions are seen to be highly similar. In fact, when subjects' individual scores are expressed in terms of gradients, the differences between the three experimental conditions are no longer statistically significant (Kruskal-Wallis $\mathrm{H}=.54, \mathrm{p}>.7$ ).

A gradient measure appears, therefore, to represent an adequate measure of the effective stimulus for curvature. It has the additional merit, as we shall show, of affording comparisons of threshold data obtained in different experiments using any lengths of spherically curved surfaces. However, it is obvious that, for any set of standard length surfaces, the gradient measure is simply a linear transformation of base-to-peak height. This latter measure is expressible in simple units and is easily visualized. For these reasons, others may prefer to use base-to-peak height (or arc height) in studies using equal length surfaces. We shall continue to use base-to-peak height in the remainder of this paper.

\section{EXPERIMENT 4: SPHERICAL VS. CYLINDRICAL CURVATURE}

In this experiment, a simple comparison was made between two types of curvature: spherical and cylindrical. The obvious advantages of using plano-convex lenses for the production of test surfaces have been outlined earlier. These spherical lenses have the additional advantage that all routes crossing the center of the curved surface have the same curvature. But spherical lenses have one disadvantage: any maskedoff strip will be curved in two dimensions. However, suitable one-dimensional curved strips may be obtained from plano-cylindrical lenses. Experiment 4 attempted to discover whether restricting curvature to one dimension has any significant effect upon the haptic detection of curvature. ${ }^{1}$

In order to hold constant such extraneous variables as practice, fatigue, and skin temperature, it was decided to test each subject using both types of curvature. A pilot study revealed that to do this in a single session using the constant method was excessively demanding. Accordingly, the comparison was effected by means of a staircase procedure.

\section{Method}

Apparatus. The one-dimensional curves were constructed from a set of 60-mm-diam plano-cylindrical lenses, each with a $2.0-\mathrm{mm}$ step edge. The lenses ranged from 0 to $+3.0 \mathrm{D}$ in $.25-\mathrm{D}$ steps. Test curves were constructed by masking off each lens to leave a $10 \times 40 \mathrm{~mm}$ central strip. The two-dimensional curves were prepared in a similar manner using the original plano-convex lenses.

Subjects. The subjects were 12 paid psychology undergraduates, with a mean age of 21 years.

Procedure. Each subject attended for a single session, lasting about $1 \mathrm{~h}$. During a session, absolute thresholds for curvature were measured using (a) the cylindrical curves and (b) the spherical curves. The ordering of (a) and (b) was counterbalanced across subjects. Thresholds were obtained using a forced-choice doublestaircase procedure (Cornsweet, 1962) consisting of 40 trials. Data from the first five trials of the ascending and descending staircases were omitted from threshold calculations. A 5-min rest was given between the two threshold determinations.

\section{Results}

The overall average thresholds, expressed as baseto-peak heights, were as follows: spherical curves, $\mathrm{M}=.22 \mathrm{~mm}(\mathrm{SD}=.10 \mathrm{~mm})$; cylindrical curves, $\mathrm{M}=$ $.25 \mathrm{~mm}(\mathrm{SD}=.09 \mathrm{~mm})$. Converted to gradient measures, the spherical and cylindrical means are equivalent to .011 and .012 , respectively-results that are quite close to those reported in the 40-mm strip length condition (average $=.009$ ) in Experiment 3 .

More importantly, however, the subjects' mean thresholds under the two conditions of curvature do not differ significantly $[t(11)=.89, \mathrm{p}>.05]$.

We conclude that type of curvature is not an important variable when narrow surfaces are used as test stimuli. This is not to deny that two-dimensional curvature may contain an important additional cue when larger surfaces are explored. This may be particularly important in more natural, real-life situations.

\section{EXPERIMENT 5: THE DIRECT SCALING OF HAPTICALLY PERCEIVED CURVATURE}

In this final experiment, an attempt was made to discover how the sensation of curvature grows as a function of objective curvature, using a direct scaling procedure. Direct scaling has been used several times in work on tactile or haptic sensitivity, most recently by Lederman (1978) in an investigation of the perception of roughness. In the present experiment, two levels of difficulty were employed. In the more diffi- 
cult condition, judgments were obtained using a relatively narrow range of curved surfaces. This range was extended in the easier condition.

\section{Method}

Apparatus. The surfaces were prepared from the same type of 60-mm-diam plano-convex lenses used in the previous experiments. Each lens was masked off to leave a smooth strip $10 \mathrm{~mm}$ wide running across the entire length of the lens. In Condition A, the 10 lenses ranged in power from .75 to $3.0 \mathrm{D}$ in .5-D steps. In terms of base-to-peak height, this range is equivalent to .65 to $2.6 \mathrm{~mm}$. In Condition $\mathrm{B}$, the 10 lenses ranged from 1.0 to 10.0 D in 1.0-D steps. In terms of base-to-peak heights, this is equivalent to .86 to $9.46 \mathrm{~mm}$.

Subjects. The subjects were 26 psychology undergraduates, with a mean age of 20 years. Fourteen subjects were tested in Condition $\mathrm{A}$, and 12 were tested in Condition B. No subject had had previous experience in a scaling task.

Procedure. This was modeled on Lederman and Taylor's (1972) description of their scaling procedure. The subject was presented with the extremes of the range of stimuli to be used and was informed that these were "one of the flattest" and "one of the most curved" surfaces to be presented. The set of 10 surfaces was then presented twice in random order. On each presentation, the subject moved the tip of the second finger of the preferred hand across the surface and back and then assigned a number to the subjective impression of curvature. Free choice of module was permitted. Talcum powder was used to reduce friction between the skin and the glass surface.

\section{Results}

The geometric means of subjects' paired ratings were transformed to reduce intersubject variability (Engen, 1972, p. 77). The transformed ratings were subjected to trend analysis. Finally, the linear functions and power functions relating overall mean ratings and surface curvature were derived.

Condition $\mathbf{A}$. The ratings obtained from the narrower range of lenses show a highly significant linear trend $[F(1,117)=13.9, p<.001]$. There is no significant quadratic trend $(F<1.0)$. The power function between ratings and curvature is $R=5.2 \mathrm{ht}^{1.2}\left(\mathrm{r}^{2}=\right.$

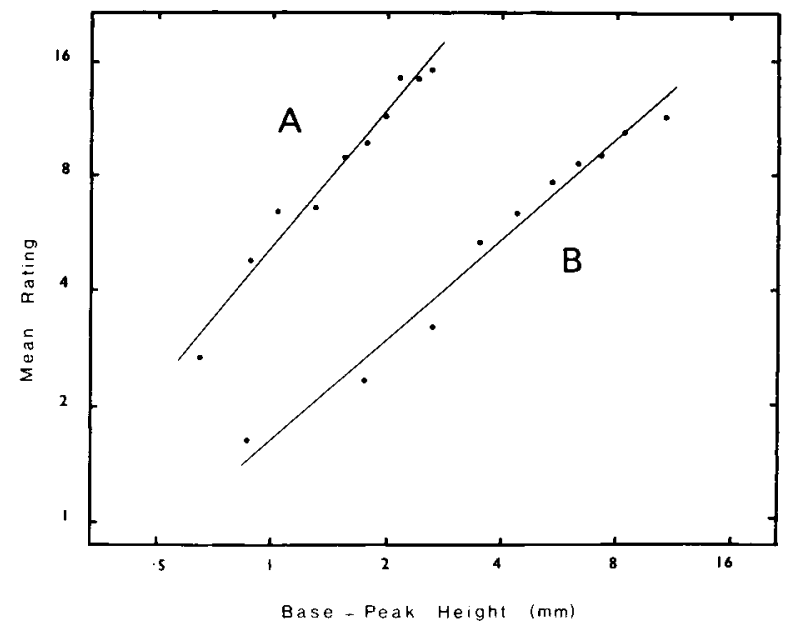

Figure 1. Mean ratings of curvature in Experiment 5, with Condition $A$ using a narrow range of curves and Condition $B$ using a wide range of curves.
.98 ), where $R=$ the overall mean of the transformed ratings, and $\mathrm{ht}=$ base-to-peak surface height in $\mathrm{mm}$. A linear function fitted to the same data has the form: $\mathrm{R}=6.6 \mathrm{ht}-1.2\left(\mathrm{r}^{2}=.98\right)$.

Condition B. The ratings obtained using the wider range of lenses also show a highly significant trend $[F(1,99)=1.3, p>.05]$. There is no significant quadratic trend. The power function between ratings and curvature in this condition is $\mathrm{R}=1.7 \mathrm{ht} \cdot{ }^{9}\left(\mathrm{r}^{2}=.98\right)$. The corresponding linear function is $R=1.2 \mathrm{ht}+.7$ $\left(r^{2}=.98\right)$. Figure 1 shows the relationship between ratings and curvature, plotted in the conventional $\log -\log$ form.

\section{GENERAL DISCUSSION}

We have stated that the ability to detect curvature and differences in curvature appears to us to be impressive. The question arises as to whether the present threshold data are better than those obtain ed by previous workers, commonly via the use of much longer curved surfaces.

There are certain difficulties in attempting comparisons between the present results and others published previously. In the early study by Rubin (1936), for example, the question asked was what degree of curvature could be imparted to a flexible steel strip while the edge continued to feel straight. Rubin presents data on the threshold for straightness ("Geradheitszreiz') and on the threshold for curvature ("Schwellenwert"), but no reliability estimates are given. Similar criticisms apply to the work of Crewdson and Zangwill (1940). Hunter's (1954) thresholds cannot be computed from his table of results.

An additional, if minor, source of difficulty is caused by the nature of the curved surfaces used by Crewdson and Zangwill (1940) and Rubin (1936). Displacing the center of a flexible strip to produce curvature requires that one end of the strip be free to move. But such an arrangement generates curves that are not symmetrical; they are, in fact, cubic polynomials, as Rubin acknowledged. This criticism does not apply to Davidson's (1972) experiment, in which rigid curved surfaces were used, although, as stated earlier, reliability data are not included in his report.

An attempt was made to compare the present results on absolute thresholds for curvature with those from three earlier studies. We used the crude but simple procedure of calculating, using published threshold data, the gradients of the test surfacesthat is, by dividing the base-to-peak heights at threshold by half the length of the surfaces. In cases in which there was an important choice, we have used the lowest threshold data available: for example, we use only data from the "long arm" condition in the Crewdson and Zangwill (1940) study. This simple conversion to threshold gradients yields the following results: Rubin (1936), threshold gradient $=.014$; 
Crewdson and Zangwill (1940), threshold gradient $=$ .017; Davidson (1972), threshold gradients $=.013$ (blind subjects) and .017 (sighted subjects); Gordon and Morison (present study, Experiment 1), threshold gradient $=.009$. We conclude that the perception of curvature is most sensitive when scanning movements are small and do not involve movement of the forearm.

The final part of this report dealt with the subjective response to curvature. Previous studies in the general area of tactile perception have reported that the relationship between stimulus magnitude and subjective response is often best described by a power function. Power function relationships have been demonstrated in tactile responses to vibration (Stevens, 1959a), electric shock (Stevens, 1959b), and roughness (Lederman \& Taylor, 1972; Stevens \& Harris, 1962). Although we have fitted power functions to the data from Experiment 5 and have used the conventional $\log -\log$ plots in Figure 1, it is clear that the results show strong linear trends: in fact, the linear functions in both conditions fit the data as well as the power functions do. This result was unexpected.

Although there has been controversy concerning the nature and interpretation of the "power law" (e.g., Treisman, 1964), the empirical evidence from the various studies of tactile perception listed above does demonstrate that power function relationships are found in this modality. The response to curvature may be exceptional because curvature is a metathetic rather than a prothetic dimension, but this is a description rather than an explanation. However, we note that there is a precedent for our finding: the response to intensity of tactile vibration at $60 \mathrm{~Hz}$ yields a power function having an exponent of approximately 1.0 (Stevens, 1968).

In future research, we shall attempt to assess the relative contribution of joint vs. pressure receptors in the perception of curvature, and the differences, if any, between active and passive scanning. We are currently working with blind people in order to see whether their lengthy and intensive tactile experience leads to superior performance at the threshold tasks we have described.

\section{REFERENCES}

Connsweet, T. N. The staircase method in psychophysics. American Journal of Psychology, 1962, 75, 485-491.

Crewdson, J., \& Zangwill, O. L. A note on tactual perception in a blind subject. British Journal of Psychology, 1940, 30, 224-229.

Davidson, P. W. Haptic judgments of curvature by blind and sighted humans. Journal of Experimental Psychology, 1972, 93, 43-55.

ENGEN, T. Psychophysics. In J. W. Kling \& L. A. Riggs (Eds.), Woodworth \& Schlosberg's experimental psychology. London: Methuen, 1972.

Gordon, I. E., \& Cooper, C. Improving one's touch. Nature, 1975, 256, 203-204.

Hunte R, I. M. L. Tactile-kinesthetic perception of straightness in blind and sighted humans. Quarterly Journal of Experimental Psychology, 1954, 6, 149-154.

LEDERMAN, S. J. Heightening tactile impressions of surface texture. In G. Gordon (Ed.), Active touch. Oxford: Pergamon Press, 1978.

Lederman, S. J., \& Taylor, M. M. Fingertip force, surface geometry, and the perception of roughness by active touch. Perception \& Psychophysics, 1972, 12, 401-408.

Rubin, E. Haptische Untersuchungen. Acta Psychologica, 1936, $1,285-380$.

StEvens, S. S. Tactile vibration: Dynamics of sensory intensity. Journal of Experimental Psychology, 1959, 57, 210-218. (a)

Stevens, S. S. Cross-modality validation of subjective scales for loudness, vibration, and electric shock. Journal of Experimental Psychology, 1959, 57, 201-209. (b)

Stevens, S. S. Tactile vibration: Change of exponent with frequency. Perception \& Psychophysics, 1968, 3, 223-228.

Stevens, S. S., \& Harris, J. R. The scaling of subjective roughness and smoothness. Journal of Experimental Psychology, $1962,64,489-494$.

Treisman, M. Sensory scaling and the psychophysical law. Quarterly Journal of Experimental Psychology, 1964, 16, 11-22.

\section{NOTE}

1. This experiment was suggested by an anonymous reviewer.

(Manuscript received July 29, 1981;

revision accepted for publication January 20, 1982.) 